\title{
Supine-Related Pseudoanemia in Hospitalized Patients
}

\author{
Arsalan Derakhshan, MD*, Reza Manesh, MD², Bennett A Peterson, MD³, Bibhu D Mohanty, MD, \\ Thomas S Kickler, MD5, Daniel J Brotman, $\mathrm{MD}^{6}$
}

\begin{abstract}
'Department of Internal Medicine, Case Western Reserve University School of Medicine, University Hospital Cleveland Medical Center, Cleveland, Ohio; ${ }^{2}$ Department of Internal Medicine, Johns Hopkins University School of Medicine, Baltimore, Maryland; ${ }^{3}$ Department of Internal Medicine, Saint Joseph's Medical Center, Towson, Maryland; ${ }^{4}$ Division of Cardiology, Department of Medicine, University of South Florida, Morsani College of Medicine, Tampa, Florida; ${ }^{5}$ Department of Pathology, Johns Hopkins University School of Medicine, Baltimore, Maryland; ${ }^{6}$ Department of Medicine, Johns Hopkins University School of Medicine, Baltimore, Maryland.
\end{abstract}

A patient's supine posture redistributes plasma into the vascular space, leading to dilution of blood constituents. The extent to which posture may influence identification of hospital-acquired anemia is unknown. Patients in this quasi-experimental study had blood obtained for hemoglobin measurement while recumbent for at least 6 hours, and then again after sitting upright for at least 1 hour. Of the 35 patients who completed the study, 13 were women (37\%). Patients had a median increase in hemoglobin of $0.60 \mathrm{~g} / \mathrm{dL}$ (range, -0.6 to $1.4 \mathrm{~g} / \mathrm{dL}$ ) with sitting, a $5.2 \%$ (range, $(-4.5 \%$ to $15.1 \%$ ) relative change $(P<.001)$. Ten of 35 patients $(29 \%)$ exhibited an increase in hemoglobin of $1.0 \mathrm{~g} / \mathrm{dL}$ or more. Posture influences hemoglobin levels in hospitalized patients on general medicine wards; this knowledge may help curb unnecessary testing to evaluate small changes in hemoglobin concentration. Journal of Hospital Medicine 2021;16:219-222. (C) 2021 Society of Hospital Medicine

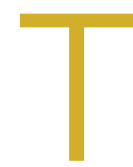

he World Health Organization (WHO) defines anemia as a hemoglobin value less than $12 \mathrm{~g} / \mathrm{dL}$ in women and less than $13 \mathrm{~g} / \mathrm{dL}$ in men. ${ }^{1}$ Hospital-acquired anemia is loosely defined as normal hemoglobin levels on admission that, at their nadir during hospitalization or on discharge, are less than WHO sex-defined cutoffs. Hospitalacquired anemia or significant decreases in hemoglobin are often identified during hospitalization. ${ }^{2-6}$ Potential causes include blood loss from phlebotomy, occult gastrointestinal bleeding, hemolysis, anemia of inflammation, and hemodilution due to fluid resuscitation. Of these causes, some are dangerous to patients, some are iatrogenic, and some are due to laboratory error. ${ }^{7}$ Physicians often evaluate decreases in hemoglobin, which could otherwise be explained by laboratory error, hemodilution, or expected decrease in hemoglobin due to hospitalization, to identify causes that may lead to potential harm.

Jacob et $\left.a\right|^{8}$ demonstrated the effect of posture on hemoglobin concentrations in healthy volunteers, showing an average $11 \%$ relative increase in hemoglobin when going from lying to standing. This increase was attributed to shifts in plasma volume to the vascular space with recumbence. They hypothesized that the initial hemoglobin on admission is measured when patients are upright or recently upright, whereas after

*Corresponding Author: Arsalan Derakhshan, MD; Email: Arsalan.Derakhshan @UHhospitals.org; Twitter: @ArsalanMedEd.

Published online first March 17, 2021.

Received: August 11, 2020; Revised: November 28, 2020;

Accepted: November 29, 2020

(C) 2021 Society of Hospital Medicine DOI 10.12788/jhm.3580 admission, patients are more likely to be supine, resulting in lower hemoglobin concentrations. Others have also demonstrated similar effects of patient posture on hemoglobin concentration. ${ }^{9-13}$ However, these prior results are not readily generalizable to hospitalized patients. These prior studies enrolled healthy volunteers, and most examined postural changes from the supine and standing positions; blood is rarely obtained from hospitalized patients when they are standing.

The aim of this study was to investigate whether postural changes in hemoglobin can be demonstrated in positions that patients routinely encountered during in-hospital phlebotomy: upright in a chair or recumbent in a bed. Patient position, which is not standardized during blood draws, may contribute to lower measured hemoglobin concentrations in some patients, especially sicker individuals who are recumbent more frequently. We hypothesized that going from supine to upright in a chair would result in a relative increase in hemoglobin concentration of $5 \%$ to $6 \%$, approximately half the value of going from supine to standing. ${ }^{8}$ To investigate this, we conducted a quasi-experimental study exploring the effect of position (supine or sitting in chair) on hemoglobin concentrations in medical inpatients.

\section{METHODS}

\section{Participants}

Patients were enrolled in this single-center study between October 2017 and August 2018. Patients aged 18 years or older who were hospitalized on the general internal medicine wards were screened to determine if they met the following inclusion criteria: hospitalized for $<5$ days, had blood work scheduled as part of routine care (in order to decrease phlebotomy required by this study), had baseline hemoglobin $>8 \mathrm{~g} / \mathrm{dL}$, and 

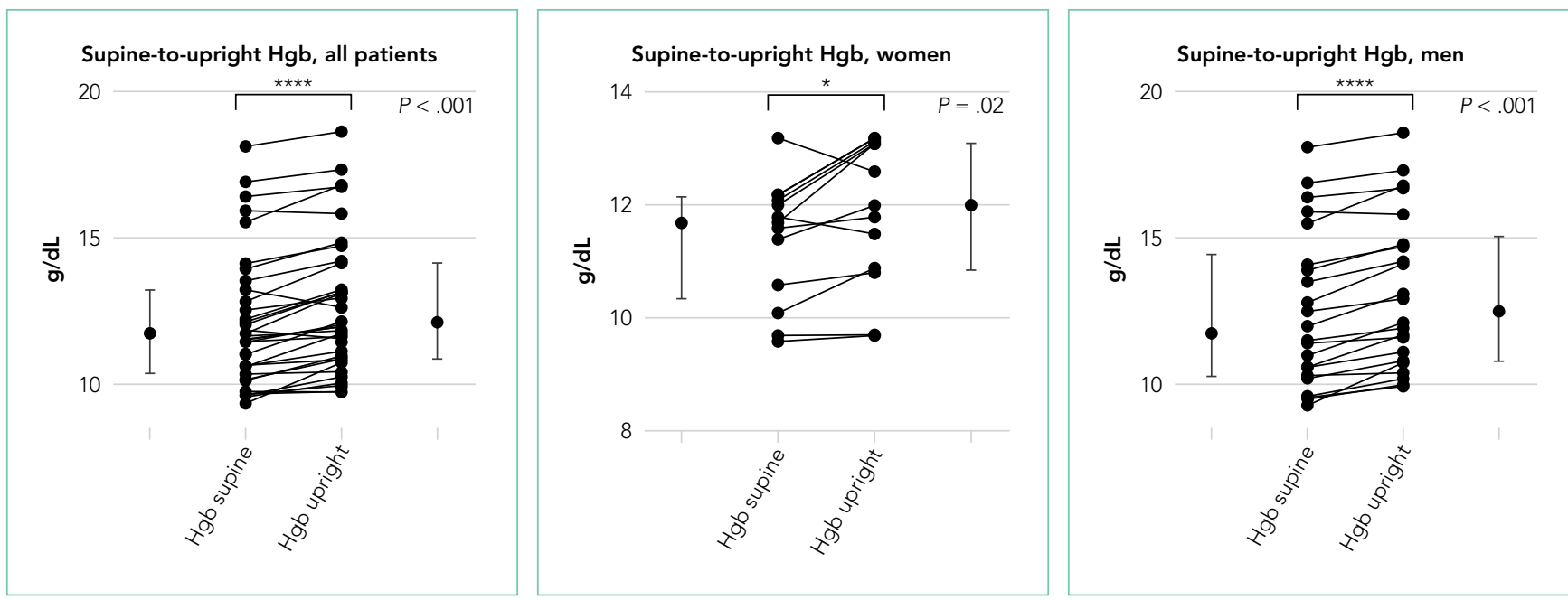

FIG 1. Patient-Level Hemoglobin Changes With Posture Changes. The before-after plots flanked by respective median values and interquartile ranges show the effects of going from a supine to an upright seated position on hemoglobin ( $\mathrm{Hgb}$ ) concentrations in all patients (women, $\mathrm{n}=13 ;$ men, $\mathrm{n}=22$ ). Each line represents one studied participant ( $\mathrm{N}=35)$.

were able to remain supine without interruption overnight and able to sit in a chair for at least 1 hour the following morning. Patients were excluded from the study if they had a hematologic malignancy, were at risk of $>100 \mathrm{~mL}$ of blood loss (eg, admitted for gastrointestinal bleeding, planned surgery), had a transfusion requirement, or received intravascular modifiers such as fluid (>100 cc/h) or intravenous diuretics. The Johns Hopkins Institutional Review Board approved this study, and all patients provided written informed consent.

\section{Study Design}

Patients enrolled in this quasi-experimental study were asked to remain supine for at least 6 hours overnight. Adherence to the recumbent position was tracked by patient self-report and by corroboration with the patient's nurse overnight. Any interruptions to supine positioning resulted in exclusion from the study. The following morning, a member of the study team performed phlebotomy while the patient remained supine. Patients were then asked to sit comfortably in a chair for at least 1 hour with their feet on the ground; the blood draw was then repeated. All blood samples were acquired by venipuncture. Prior to each blood draw, a tourniquet was placed over the upper arm below the axilla. An antecubital vein on either arm was visualized under ultrasound guidance, and a 23-G $\times 3 / 4$ " butterfly needle was used for venipuncture. The vials of blood were immediately inverted after blood collection. Hemoglobin assays were processed and analyzed using Sysmex XN-10 analyzer (Sysmex Corporation). The reference range for hemoglobin in our facility was 12.0 to $15.0 \mathrm{~g} / \mathrm{dL}$ for women and 13.9 to $16.3 \mathrm{~g} / \mathrm{dL}$ for men. Laboratory technicians were blinded to and uninvolved in the study.

We determined, a priori, that 33 enrolled patients would provide $80 \%$ power (alpha 0.05 ) to detect an average hemoglobin change of $4.1 \%$, assuming that the standard deviation of the hemoglobin change was twice the mean (ie, SD $=8.2 \%$ ). The Wilcoxon signed-rank test was used to test the significance of postural hemoglobin changes. Analyses were conducted using JMP Pro 13.0 (SAS) and GraphPad Prism 8 (GraphPad Software). Significance was defined at $P<.05$ for all analyses.

\section{RESULTS}

Thirty-nine patients were consented and enrolled in the study; four patients were excluded prior to blood draw (two patients because of interruption of supine time, two patients because of refusal in the morning). Of the 35 patients who completed the study, 13 were women (37\%); median age was 49 years (range, $25-83$ years). Median supine hemoglobin concentration in our sample was $11.7 \mathrm{~g} / \mathrm{dL}$ (range, 9.3-18.1 g/dL), and median baseline creatinine level was $0.70 \mathrm{mg} / \mathrm{dL}$ (range, $0.5-2.5 \mathrm{mg} / \mathrm{dL}$ ). Median supine hemoglobin levels were $11.7 \mathrm{~g} / \mathrm{dL}$ (range, 9.6-13.2 $\mathrm{g} / \mathrm{dL}$ ) in women and $11.8 \mathrm{~g} / \mathrm{dL}$ (range, 9.3-18.1 g/dL) in men. In aggregate, patients had a median increase in hemoglobin concentration of $0.60 \mathrm{~g} / \mathrm{dL}$ (range, -0.6 to $1.4 \mathrm{~g} / \mathrm{dL}$ ) with sitting, a $5.2 \%$ (range, $-4.5 \%$ to $15.1 \%$ ) relative change $(P<.001)$ (Figure 1 ). Women had a median increase in hemoglobin concentration of $0.60 \mathrm{~g} / \mathrm{dL}$ (range, -0.6 to $1.4 \mathrm{~g} / \mathrm{dL}$ ) with sitting, a relative change of $5.3 \%$ (range, $-4.5 \%$ to $12.0 \%)(P=.02$ ). Men had a median increase in hemoglobin concentration of $0.55 \mathrm{~g} / \mathrm{dL}$ (range, -0.1 to $1.4 \mathrm{~g} / \mathrm{dL}$ ) with sitting, a $5.0 \%$ (range, $-0.6 \%$ to $15.1 \%$ ) relative change $(P<.001)$. Ten of 35 participants $(29 \%)$ exhibited an increase in hemoglobin level of $1.0 \mathrm{~g} / \mathrm{dL}$ or more (Figure 2).

\section{DISCUSSION}

International blood collection guidelines acknowledge postural changes in laboratory values and recommend standardization of patient position to either sitting in a chair or lying flat in a bed, without changes in position for 15 minutes prior to blood draw. ${ }^{14}$ When these positional accommodations cannot be met, documenting positional disruptions is recommended so that laboratory values can be interpreted accordingly. To the best of our knowledge, no hospital in the United States has standardized patient position as part of phlebotomy procedure such that patient position is documented and can be 


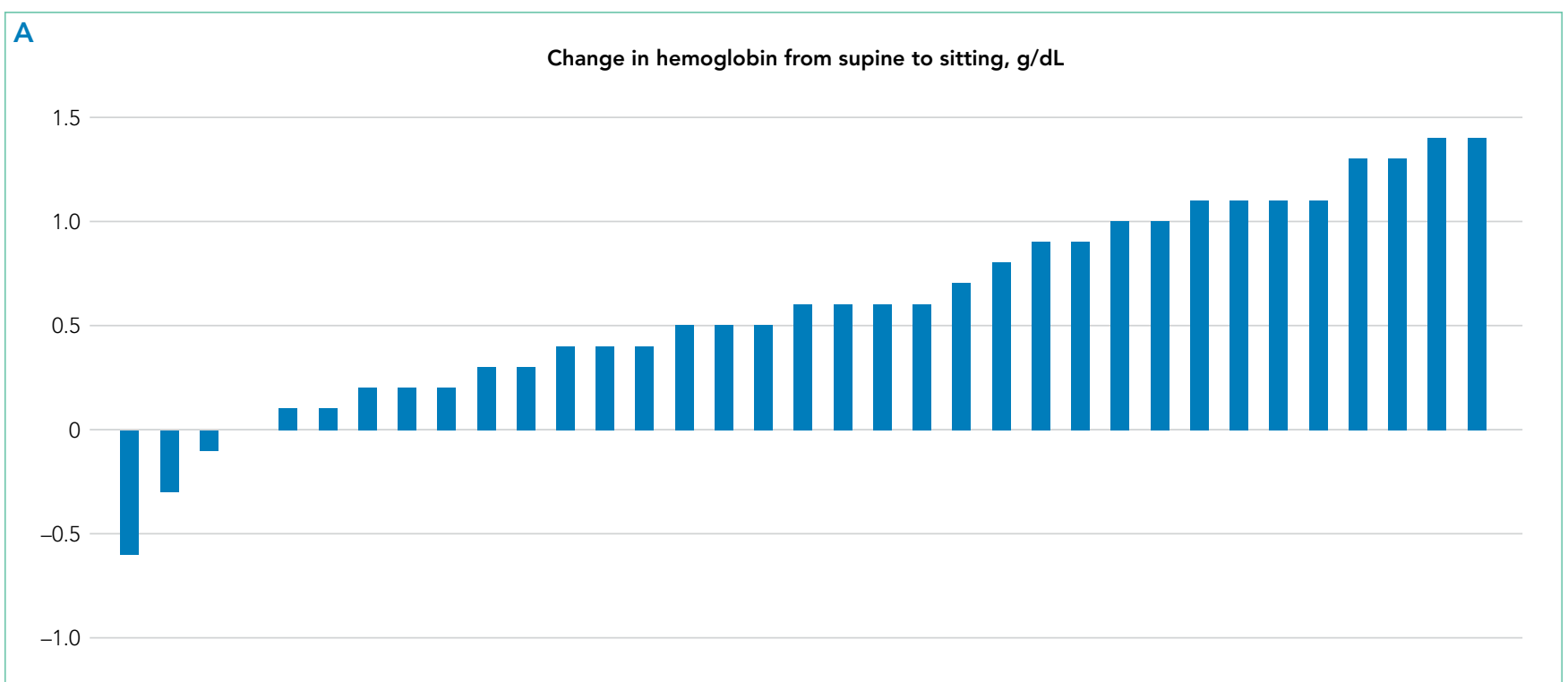

B

Change in hemoglobin from supine to sitting, \%

20.0

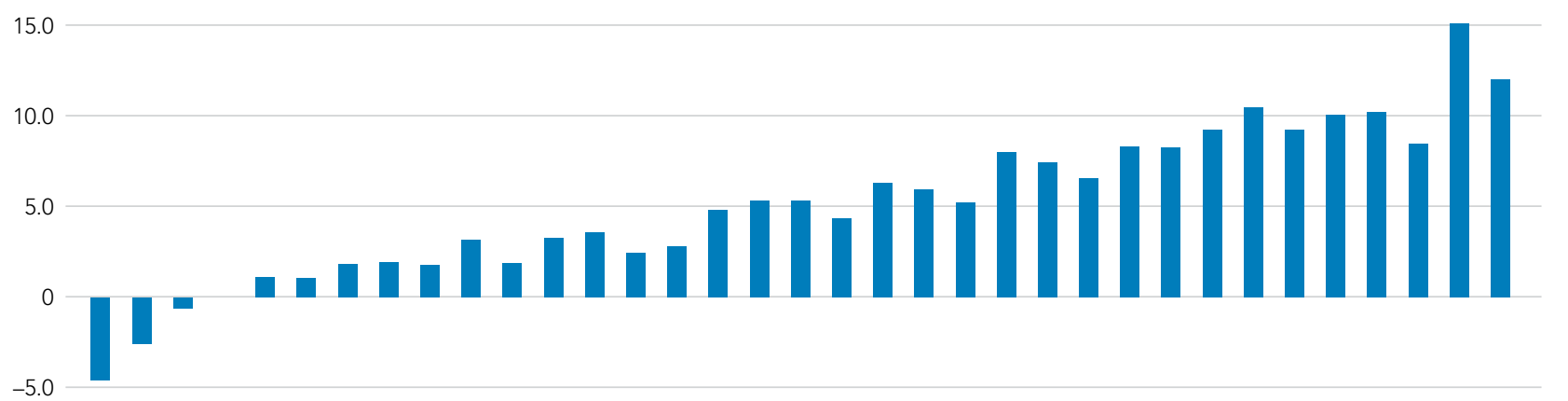

$-10.0$

FIG 2. Absolute and Relative Change in Hemoglobin Concentration With Positional Changes. The bar graphs illustrate (A) the absolute change in hemoglobin concentration $(\mathrm{g} / \mathrm{dL})$ and $(\mathrm{B})$ the percent change in hemoglobin concentration. Each bar represents one studied participant $(\mathrm{N}=35)$. Bars are aligned such that the absolute change in hemoglobin from the top graph correlates with the percent change in the bottom graph.

made available to interpreting providers.

Relative increases in hemoglobin or hematocrit range from $7 \%$ to $12 \%$ when patients go from supine to standing. $8,9,11$ The reverse relationship has also been shown, where upright-tosupine position results in decreases in hemoglobin concentrations. ${ }^{10,13}$ We found that going from supine to a seated position resulted in significant increases in hemoglobin of $0.6 \mathrm{~g} / \mathrm{dL}$ and in a more than $1 \mathrm{~g} / \mathrm{dL}$ increase in $29 \%$ of the patients. Although four of the 35 patients experienced either no change or a slight decrease in their hemoglobin concentration when going from supine to upright and not all patients saw a uniform effect, providers should be aware that the patient's position can contribute to changes in hemoglobin concentration in the hospitalized setting. Providers may be able to use this information to avoid an extensive diagnostic workup when anemia is identified in hos- pitalized patients, although more research is needed to identify patient subsets who are at higher risk for this effect.

Until hospitals implement protocols that require phlebotomists to report patient position during phlebotomy in a standardized fashion, providers should be alert to the fact that supine positioning may result in a hemoglobin level that is significantly lower than that when drawn in a sitting position, and in almost one-third of patients, this difference may be $1.0 \mathrm{~g} / \mathrm{dL}$ or greater.

Given our study criteria requiring supine positions of at least 6 hours and a baseline hemoglobin concentration $>8 \mathrm{~g} / \mathrm{dL}$, our sample of patients may have been younger and healthier than the average hospitalized patient on general internal medicine wards. Since greater relative changes in plasma volume shifts and hemoglobin might be seen in patients with lower baseline 
hemoglobin and lower baseline plasma protein, this selection bias may underestimate the effects of position on hemoglobin changes for the average inpatient population. Additionally, we intentionally sought to obtain sitting hemoglobin levels after the supine samples to avoid the possibility of incorrectly attributing dropping hemoglobin levels to progressive hospitalacquired anemia from phlebotomy or illness. Any concomitant trend of falling hemoglobin levels in our patients would be expected to lead to a systematic underestimation of the positional change in hemoglobin we observed. We did not objectively observe adherence to supine and upright position and instead relied on patient self-reporting, which is one possible contributor to the variable effects of position on hemo-

\section{References}

1. DeMaeyer $E$, Adiels-Tegman M. The prevalence of anaemia in the world World Health Stat Q. 1985;38(3):302-316.

2. Martin ND, Scantling D. Hospital-acquired anemia. J Infus Nurs. 2015;38(5):330-338. https://doi.org/10.1097/NAN.0000000000000121

3. Thavendiranathan P, Bagai A, Ebidia A, Detsky AS, Choudhry NK. Do blood tests cause anemia in hospitalized patients? The effect of diagnostic phlebotomy on hemoglobin and hematocrit levels. J Gen Intern Med. 2005;20(6):520-524. https://doi.org/10.1111/j.1525-1497.2005.0094.x

4. Salisbury AC, Reid KJ, Alexander KP, et al. Diagnostic blood loss from phlebotomy and hospital-acquired anemia during acute myocardial infarction. Arch Intern Med. 2011;171(18):1646-1653. https://doi.org/10.1001/archinternmed.2011.361

5. Languasco A, Cazap N, Marciano S, et al. Hemoglobin concentration variations over time in general medical inpatients. J Hosp Med. 2010;5(5):283-288. https://doi.org/10.1002/jhm.650

6. van der Bom JG, Cannegieter SC. Hospital-acquired anemia: the contribution of diagnostic blood loss. J Thromb Haemost. 2015;13(6):1157-1159. https://doi.org/10.1111/jth.12886

7. Berkow L. Factors affecting hemoglobin measurement. J Clin Monit Comput. 2013;27(5):499-508. https://doi.org/10.1007/s10877-013-9456-3

8. Jacob G, Raj SR, Ketch $T$, et al. Postural pseudoanemia: posture-dependent change in hematocrit. Mayo Clin Proc. 2005;80(5):611-614. https:// doi.org/10.4065/80.5.611 globin concentration, with some patients having no change or decreases in hemoglobin concentrations.

\section{CONCLUSION}

Posture can significantly influence hemoglobin levels in hospitalized patients on general medicine wards. Further research can determine whether it would be cost and time effective to standardize patient positions prior to phlebotomy, or at least to report patient positioning with the laboratory testing results.

Disclosures: The authors have no financial relationships or conflicts of interest relevant to this article to disclose.

9. Fawcett JK, Wynn V. Effects of posture on plasma volume and some blood constituents. J Clin Pathol. 1960;13(4):304-310. https://doi.org/10.1136 /jcp.13.4.304

10. Tombridge TL. Effect of posture on hematology results. Am J ClinPathol. 1968;49(4):491-493. https://doi.org/10.1093/ajcp/49.4.491

11. Hagan RD, Diaz FJ, Horvath SM. Plasma volume changes with movement to supine and standing positions. J Appl Physiol. 1978;45(3):414-417. https:// doi.org/10.1152/jappl.1978.45.3.414

12. Maw GJ, Mackenzie IL, Taylor NA. Redistribution of body fluids during postural manipulations. Acta Physiol Scand. 1995;155(2):157-163. https:// doi.org/10.1111/j.1748-1716.1995.tb09960.x

13. Lima-Oliveira G, Guidi GC, Salvagno GL, Danese E, Montagnana M, Lippi G. Patient posture for blood collection by venipuncture: recall for standardization after 28 years. Rev Bras Hematol Hemoter. 2017;39(2):127-132. https:// doi.org/10.1016/j.bjhh.2017.01.004

14. Simundic AM, Bölenius K, Cadamuro J, et al. Working Group for Preanalytical Phase (WG-PRE), of the European Federation of Clinical Chemistry and Laboratory Medicine (EFLM) and Latin American Working Group for Preanalytical Phase (WG-PRE-LATAM) of the Latin America Confederation of Clinical Biochemistry (COLABIOCLI). Joint EFLM-COLABIOCLI recommendation for venous blood sampling. Clin Chem Lab Med. 2018;56(12):2015-2038. https://doi.org/10.1515/cclm-2018-0602 